\title{
A promising line of defence
}

Each year, mosquito-borne infectious diseases such as malaria and dengue fever cause millions of deaths worldwide. Olfactory cues that are emitted from breath, skin and sweat attract female blood-feeding mosquitoes to vertebrate hosts. $\mathrm{CO}_{2}$ that is present in breath is a key attractive cue for mosquitoes. Reporting in Nature, Ray and colleagues show that compounds that interfere with the insects' $\mathrm{CO}_{2}$-detection machinery disrupt host-seeking behaviour and might be used to develop a new generation of mosquito traps and repellents.

$\mathrm{CO}_{2}$ is detected by heteromeric receptors that are highly conserved across dipterans, including Drosophila spp. The authors took a panel of compounds that are structurally related to odorants that inhibit the electrophysiological and behavioural responses to $\mathrm{CO}_{2}$ in D. melanogaster and looked for odorants that affected the $\mathrm{CO}_{2}$-sensitive neurons in three major species of disease-transmitting mosquitoes: Anopheles gambiae, Culex quinquefasciatus and A. aegypti.
One of the compounds that they identified - 2,3-butanedione - caused an unusually prolonged activation of $\mathrm{CO}_{2}$-detecting neurons in all three mosquito species. Unlike the response to $\mathrm{CO}_{2}$, which decayed immediately after the end of the stimulus, the neuronal response to a 1-s pulse of 2,3-butanedione lasted for over 5 minutes. Importantly, during this time the neurons were less responsive to $\mathrm{CO}_{2}$ stimulation, suggesting that the $\mathrm{CO}_{2}$-mediated attraction behaviour of female mosquitoes could be disrupted. Using video tracking in a wind tunnel, the authors showed that pre-exposure to a blend of four odorants (2,3-butanedione, 1-hexanol, 1-butanal and 1-pentanal) caused prolonged activation of $\mathrm{CO}_{2}$-detecting neurons and impaired the mosquitoes' ability to detect and find a $\mathrm{CO}_{2}$ source in a dose- and time-dependent manner. Furthermore, when they examined hut-entry behaviour in semi-field conditions, dispersion of the odorant blend resulted in fewer

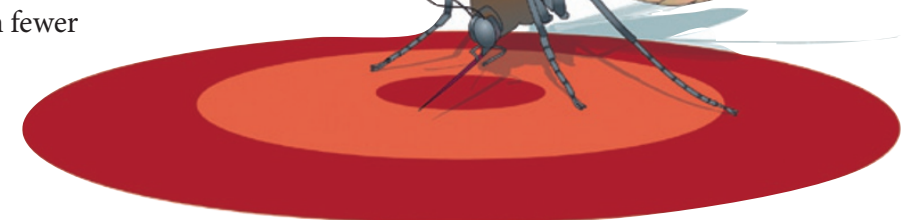

mosquitoes being attracted to $\mathrm{CO}_{2}$ traps inside the huts.

Together, these findings suggest that prolonged activation of the $\mathrm{CO}_{2}$-sensitive neurons in mosquitoes might be a useful strategy in reducing mosquito-human contact. Although 2,3-butanedione has an undesirable safety profile at high concentrations and is not ideal for human use, it could lead to the development of new insect repellents that overcome the shortfalls of existing ones such as $N, N$-diethyl-meta-toluamide (DEET), which requires repeated application directly to the skin and has led to the emergence of resistant mosquito populations.

Monica Hoyos Flight

ORIGINAL RESEARCH PAPER Turner, S. L. et al. Ultra-prolonged activation of $\mathrm{CO}_{2}$-sensing neurons disorients mosquitoes. Nature. 474, 87-91 (2011)

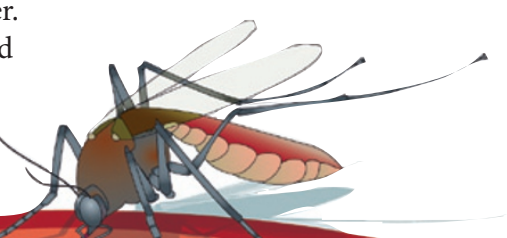

